Published in final edited form as:

Curr Opin Rheumatol. 2016 May ; 28(3): 228-235. doi:10.1097/BOR.0000000000000271.

\title{
Retinal Vasculitis
}

\author{
James T. Rosenbaum ${ }^{1,2,3,4}$, Cailin H. Sibley ${ }^{2}$, and Phoebe Lin ${ }^{1}$ \\ ${ }^{1}$ Department of Ophthalmology, Casey Eye Institute, Oregon Health \& Sciences University, \\ Portland, Oregon 97239 \\ ${ }^{2}$ Department of Medicine, Oregon Health \& Sciences University \\ ${ }^{3}$ Department of Cell Biology, Oregon Health \& Sciences University \\ ${ }^{4}$ Legacy Devers Eye Institute, Legacy Health System, Portland, Oregon 97210
}

\begin{abstract}
Purpose of review-Ophthalmologists and rheumatologists frequently miscommunicate in consulting on patients with retinal vasculitis. This report seeks to establish a common understanding of the term, retinal vasculitis, and to review recent papers on this diagnosis.

Recent findings-1) The genetic basis of some rare forms of retinal vascular disease have recently been described. Identified genes include CAPN5, TREX1, and TNFAIP3; 2) Behçet's disease is a systemic illness that is very commonly associated with occlusive retinal vasculitis; 3 ) retinal imaging including fluorescein angiography and other newer imaging modalities has proven crucial to the identification and characterization of retinal vasculitis and its complications; 4) although monoclonal antibodies to IL-17A or IL-1 beta failed in trials for Behçet's disease, antibodies to TNF alpha, either infliximab or adalimumab, have demonstrated consistent benefit in managing this disease. Interferon treatment and B cell depletion therapy via rituximab may be beneficial in certain types of retinal vasculitis.
\end{abstract}

Summary-Retinal vasculitis is an important entity for rheumatologists to understand. Retinal vasculitis associated with Behçet's disease responds to monoclonal antibodies that neutralize TNF, but the many other forms of non-infectious retinal vasculitis may require alternate therapeutic management.

\section{Keywords}

retinal vasculitis; Behçet's disease; fluorescein angiography

\section{INTRODUCTION}

Few terms create as much misunderstanding as retinal vasculitis. A rheumatologist classifies vasculitis on the basis of the size of the vessel affected, its location, and the associated histological changes. Usually this classification requires a biopsy; occasionally the diagnosis

Corresponding author: James T. Rosenbaum, Phone: 503494 5023, ; Email: rosenbaj@ohsu.edu

Conflict of interest: JTR consults for Genentech, Xoma, Novartis, Abbvie, and Janssen. The other authors report no conflict of interest. 
is based on imaging such as an abdominal angiogram. A diagnosis of vasculits indicates documented or presumed damage of the vessel wall.

In contrast, an ophthalmologist diagnoses retinal vasculitis on the basis of perivascular infiltrates on a dilated eye examination and imaging usually in the form of a fluorescein angiogram. Vasculitis can be suggested by a finding such as an intraretinal hemorrhage, which indicates an abnormality of a retinal vessel, or a cotton wool spot indicative of local retinal ischemia. A biopsy is a rarity due to the potential damage caused by a retinal biopsy. Angiography is consistent with retinal vasculitis if fluorescein stains the vessel wall or leaks beyond the vessel. This leakage demonstrates increased vascular permeability, which is a very different threshold for diagnosing vasculitis compared to vessel wall destruction on histopathology.

This review focuses on new developments in the understanding and treatment of retinal vasculitis. However, before summarizing those papers, we begin with a summary of some basic concepts relative to retinal vasculitis so that the reader will be armed with the background to appreciate the recent literature. These concepts include:

1)

Retinal vasculitis can be classified into descriptive subsets. The location of the affected retinal vessels is a critical discriminator since a vasculitis affecting vessels near the macula often affects visual acuity while a vasculitis of peripheral retinal vessels can be asymptomatic. In addition, it can be classified into occlusive or nonocclusive forms. In the occlusive form, retinal ischemia can lead to neovascularization which predisposes to vitreous hemorrhage. Retinal vasculitis can also be categorized based on whether or not it is retinal arteriole- or venule-predominant (or involving both types of retinal vessels). This distinction can be helpful to determine its etiology (Table 1). Some causes of occlusive retinal vasculitis include the vasculopathy of Susac's syndrome, IRVAN (Idiopathic Retinal Vasculitis, Aneurysms, and Neuroretinitis) ${ }^{1}$, the vasculopathy associated with anti-phospholipid antibody syndrome and systemic lupus erythematosus. A common cause of predominant retinal phlebitis is sarcoidosis. The extent of retinal vascular leakage in retinal vasculitis can also vary, from diffuse involvement, to isolated peripheral vascular leakage; the extent and location of retinal vascular involvement can be associated with ocular complications that can affect the vision.

2)

Many infections cause retinal vasculitis. These infections include viruses like cytomegalovirus, herpes simplex, human immunodeficiency virus I ${ }^{2}$ and varicella zoster; syphilis; tuberculosis; and toxoplasmosis. If an immunosuppressed patient with systemic vasculitis develops a retinal vasculitis, an infection is statistically much more common than the underlying disease as a cause of the vasculitis ${ }^{3}$. 
3)

4)

5)

6)

Retinal vasculitis in association with uveitis is common. For example, retinal vasculitis is frequently found in association with Vogt-Koyanagi-Harada syndrome or Birdshot Choroidopathy. A primary retinal vasculitis in which the uveitis does not fit into a diagnosable pattern and in which the vasculitis predominates as the clinical manifestation is well-described, but relatively uncommon ${ }^{3}$. In our own series of 207 patients with retinal vasculitis, $17 \%$ could be considered as having primary retinal vasculitis ${ }^{3}$

Behçet's disease is the rheumatic disease most consistently associated with retinal vasculitis. Other systemic diseases that commonly cause retinal vasculitis such as sarcoidosis and multiple sclerosis are not known to cause a systemic vasculitis.

With the exception of Behçet's disease, systemic forms of vasculitis such as granulomatosis with polyangiitis ${ }^{4}$, eosinophilic granulomatosis with polyangiitis ${ }^{5}$, microscopic polyangiitis, giant cell arteritis, polyarteritis nodosa, leukocytoclastic vasculitis, or primary angiitis of the central nervous system ${ }^{6}$ \} cause retinal vasculitis so rarely that publications are limited to case reports or very small case series.

Therapeutic choices for retinal vasculitis are affected by the cause of the vasculitis, the severity of the vasculitis, and the preferences of the patient and the provider. For example, a retinal vasculitis secondary to an infection requires treatment of the underlying infectious cause. A mild retinal vasculitis with changes mostly in the peripheral retina that minimally affect vision might be followed by observation alone with no therapeutic intervention. Severe retinal vasculitis secondary to Behcet's disease is often treated with a monoclonal antibody directed at TNF or with alpha interferon in some centers. Occlusive vasculopathy such as can occur with antiphospholipid antibody syndrome is treated with anti-platelet medications and/or anticoagulation. In most instances, specialists will attempt therapy with local or systemic corticosteroids and an anti-metabolite such as methotrexate before advancing to biologic therapy. Corticosteroids are the most rapidly effective while antimetabolites may take up to 3 months for benefit. Antibodies to TNF have been the primary choice among biologics to treat retinal vasculitis. Anti-TNF therapy can provide rapid benefit although not usually as rapidly as corticosteroid. If this class of medication fails, the literature provides very little guidance for additional options. The possibility of a masquerade secondary to an infection or malignancy should be reassessed. Recent publications relating to the therapy of retinal vasculitis are discussed below. The United States Food and Drug Administration has not approved any therapies specifically for retinal vasculitis. 
No study has specifically compared an anti-metabolite in combination with a TNF inhibitor to a TNF inhibitor alone in the treatment of either uveitis or retinal vasculitis. Third party payers are reluctant to approve a biologic therapy unless a patient has previously failed therapy with corticosteroid and with an antimetabolite. Some arguments favor the combination of a biologic and an anti-metabolite: potential additive effects from different mechanisms of action and reduced likelihood to generate antibodies that neutralize the biologic. Some arguments favor the use of the biologic alone: less risk of infection, less cost, less inconvenience. In many instances, the authors add anti-TNF therapy to therapy with an anti-metabolite, and then consider gradual discontinuation of the anti-metabolite therapy if complete control of ocular inflammation has been achieved for 6 to 12 months.

\section{NOVEL INSIGHTS}

Recent developments related to retinal vasculitis can be divided into (1) new insights into pathogenesis, (2) epidemiology and complications, (3) imaging, and (4) treatment.

\section{New insights into pathogenesis}

Very rare genetic causes of retinal vascular inflammatory disease are now being recognized and may help better elucidate the pathogenesis of certain types of retinal vasculitis. ADNIV (autosomal dominant neovascular inflammatory vitreoretinopathy), a condition that results in anterior chamber and vitreous inflammation, as well as retinal and iris neovascularization, is caused by mutations in Calpain $5^{7}$, a calcium-dependent cysteine protease coded by the CAPN5 gene. A mouse model of uveitis resulting from a mutation in calpain 5 has been described ${ }^{8}$. Several different mutations of calpain 5 can result in ophthalmic disease ${ }^{7}$. The neovascularization that occurs in ADNIV might be secondary to ischemia rather than an intrinsic disease of retinal vasculature. In addition to ADNIV, another inherited retinal vasculopathy is caused by mutations in TREX1, an exonuclease ${ }^{9}$. Polymorphisms in TREX1 have been associated with predisposition to systemic lupus erythematosus (SLE) ${ }^{10}$. Mutation in TREX1 can cause abnormal retinal and cerebral vasculature and retinal ischemia. The syndrome has been labelled retinal vasculopathy with cerebral leukodystrophy ${ }^{9}$. Mutations of the same gene cause Aicardi-Goutieres Syndrome ${ }^{11}$. A novel Behçet's-like autoinflammatory disease was recently reported due to mutations in TNFAIP3 (tumor necrosis factor alpha-induced protein 3) leading to A20 haploinsufficiency and increased expression of NF- $\kappa \mathrm{B}-$ mediated inflammatory cytokines ${ }^{12}$. Patients present early in life with oral ulcers, pathergy, dermal abscesses, chorioretinal scarring, and macular fibrosis secondary to retinal vasculitis.

In 2015, case reports and small series added to the differential diagnosis for retinal vasculitis. It was reported to occur subsequent to vaccination for influenza 1 and in a patient who had both malaria and Dengue Fever ${ }^{13}$. Intravitreal injection of vancomycin as is done in some centers after cataract surgery has been rarely associated with retinal vasculitis ${ }^{14}$. 
Patients with birdshot chorioretinopathy ${ }^{15}$ and uveitis in association with psoriasis ${ }^{16}$ were also noted to have a predisposition to retinal vasculitis.

A relatively common cause of retinal vasculitis in India is known as Eales' Disease. A recent report performed PCR for Mycobacterium tuberculosis DNA and detected it in 39\% of patients with Eales' disease ${ }^{17}$. The vasculitis is generally believed to be a hypersensitivity response to mycobacterial antigen rather than an active infection.

\section{Epidemiology and complications}

A report from Israel characterized 45 patients with retinal vasculitis ${ }^{18}$. About two thirds had an associated systemic disease. This is far higher than a report from our own center ${ }^{3}$. The difference is accounted for by the much higher prevalence of Behçet's disease in Israel since Behçet's disease accounted for more than $70 \%$ of the systemic illness among patients with retinal vasculitis.

A series of over 6000 patients with Behçet's disease from Iran concluded that $58 \%$ had eye disease and about one third had retinal vasculitis ${ }^{19}$. The slight male predominance and the relative frequency of specific organ involvement was similar in Iran to what had been reported in countries such as Turkey, Germany, and Japan.

A series of 132 patients with Behçet's disease from an eye center in Saudi Arabia found that panuveitis was the most common presentation ${ }^{20}$. The study noted that $26 \%$ of patients had retinal vasculitis at presentation.

Our own group has looked for correlations between clinical signs of retinal vasculitis or patient characteristics and outcome measures ${ }^{21}$. We found that neovascularization was more common in occlusive retinal vasculitis. We found that a complication known as an epiretinal membrane was more common in patients who had intraretinal hemorrhage or cotton wool spots as opposed to a finding known as vascular sheathing. Smoking correlated with a worse prognosis. Patients who were less than 40 years of age appeared to have more severe disease as judged by the likelihood that immunosuppressive therapy beyond corticosteroids would be prescribed as treatment. We also found that while only $1.4 \%$ of retinal vasculitis patients had systemic vasculitis, approximately $25 \%$ had some type of associated systemic disease, with sarcoidosis and Behçet's disease being the most common ${ }^{3}$. Other systemic diseases that can be associated with retinal vasculitis include Vogt-Koyanagi-Harada syndrome, multiple sclerosis, psoriatic arthritis and inflammatory bowel disease based our findings.

\section{Imaging}

As alluded to above, retinal vasculitis is generally defined by ophthalmologists as a disruption in the blood-retinal barrier as noted by retinal vascular leakage on fluorescein angiography and/or perivascular infiltrates on dilated fundus examination, usually in the presence of other signs of intraocular inflammation such as infiltrating leukocytes into the vitreous, anterior chamber, retina or choroid. Various ophthalmic imaging modalities aid in the identification of several features of retinal vasculitis including: extent and location of retinal vascular leakage (ultra widefield-fluorescein angiography); location and extent of retinal or choroidal lesions (fundus photography, optical coherence tomography or OCT, 
widefield autofluorescence); and presence of complications associated with retinal vasculitis including cystoid macular edema and retinal neovascularization (OCT and Ultra widefieldfluorescein angiography). For instance, in a patient with Behçet's disease associated retinal vasculitis with untreated disease, the extent of retinal infiltration, retinal vasculitis, and associated cystoid macular edema can be illustrated readily without retinal biopsy on ultra widefield fluorescein angiography, fundus imaging, and OCT (Figure 1). The diagnosis of Behçet's disease in this patient was made after presentation with panuveitis, oral ulceration and a pustular rash (Figure 1), all of which resolved with treatment with oral prednisone, azathioprine, and infliximab (Figure 2).

Recent imaging findings in retinal vasculitis include the association of retinal vasculitis with suprachoroidal fluid on enhanced depth imaging OCT in patients with Birdshot choroidopathy ${ }^{22}$. Errera et al. recently reported that adaptive optics imaging in retinal vasculitis detected perivascular infiltrates not seen on other standard imaging modalities such as fluorescein angiography and fundus photography ${ }^{23}$. Mesquida et al. demonstrated retinal vasculitis on ultra widefield-fluorescein angiography that was not otherwise clinically evident in Behçet's disease in 28 of 33 eyes (84.8\%), with the most common angiographic finding being diffuse vascular leakage ${ }^{24}$.

In some uveitis patients, peripheral vascular leakage is isolated (without diffuse vascular leakage), but the significance of this finding remains unclear. Our group found that peripheral vascular leakage on ultra widefield-fluorescein angiography occurred at a higher prevalence in patients thought to be clinically active compared to those with well-controlled disease ( $\mathrm{p}=0.001$ ), and that complications such as macular edema and optic disc leakage were associated with peripheral vascular leakage ${ }^{25}$. Another emerging imaging modality, OCT angiography, is able to quantitate flow within blood vessels without the use of intravenous fluorescein dye such as that used in fluorescein angiography. Using OCT angiography, we find that retinal vascular flow is diminished in retinal vasculitis patients compared to age-similar normal subjects (Investigative Ophthalmology and Visual Science, 2015;56(7): 3359 (abstract)).

\section{Treatment of non-infectious retinal vasculitis}

The treatment for retinal vasculitis depends on the cause of the vasculitis and its severity. In the setting of an infection, retinal vasculitis should be treated by managing the underlying infection. Retinal vasculitis secondary to anti-phospholipid antibodies might require anticoagulation. Some forms of retinal vasculitis cause minimal disruption of visual function and do not require therapy.

While initial results were promising ${ }^{26,27}$, biologics directed at either IL-1 beta or IL-17A, have recently failed to meet the primary endpoint in clinical trials for uveitis in general and for Behçet's disease specifically ${ }^{28,29}$. (The failure of neutralization of IL-1 beta in the treatment of Behcet's disease is based on a press release from July, 2015; https:// www.google.com/webhp?sourceid=chromeinstant\&ion=1\&espv=2\&ie=UTF-8\#q=gevokizumab\%20uveitis). 
Adalimumab was studied by Abbvie in two randomized, controlled trials for uveitis (not specifically retinal vasculitis), VISUAL I and VISUAL II. Both trials have been reported in abstract form at the 2015 annual meeting of the American College of Rheumatology. In the first trial, active uveitis was studied. The second trial enrolled patients with controlled inflammation who were oral corticosteroid dependent. In both trials, the primary endpoint was time to treatment failure. Both trials met their primary endpoint. All immunomodulatory therapy approved by the US Food and Drug Administration to date represents a form of corticosteroid. Abbvie will seek vision threatening, non-infectious uveitis as an indication for the use of adalimumab. If successful, rheumatologists would have improved ease to prescribe adalimumab for retinal vasculitis. The inhibition of TNF has been extremely successful in the management of Behçet's disease and associated retinal vasculitis. In Japan, regulatory authority has approved infliximab specifically for the treatment of Behçet's disease and recent American Uveitis Society recommendations state that "infliximab and adalimumab can be considered as first-line immunomodulatory agents for the treatment of ocular manifestations of Behçet's disease",30.

Several recent studies from different ethnic cohorts have addressed the long-term effects of TNF inhibition in Behçet's ocular disease: the first from a Spanish registry ${ }^{31}$ and the second from a Japanese registry ${ }^{32}$. In the Spanish study, 124 patients with uveitis refractory to conventional immunosuppressants were treated with either open-label infliximab or adalimumab. Over the course of one year, ocular inflammation, visual acuity, and steroid doses rapidly improved with $67.7 \%$ of patients achieving clinical remission. The number of patients with retinal vasculitis decreased from 89 (143 eyes) to 8 (13 eyes). In the Japanese study, 164 consecutive patients treated with infliximab for more than one year were retrospectively studied. The frequency of ocular inflammatory attacks was reported in the year prior to infliximab and compared to the year after infliximab and a significant reduction was observed. Similarly, visual acuity also improved. Relapses were frequent and typically responded to higher doses of infliximab. The frequency of retinal vasculitis was not specifically stated

The efficacy of TNF inhibition for Behçet's disease has been confirmed in a retrospective study from a French registry that included 124 patients with Behçet's disease ${ }^{33}$. Of those with ophthalmic disease considered to be "severe or refractory", $96 \%$ had a complete or partial response to TNF inhibition. Several different monoclonal antibodies to TNF appeared to show comparable benefit. Interestingly, the diagnosis of retinal vasculitis was associated with a statistically significant reduction in responsiveness, odds ratio 0.33 , range 0.12 to 0.89 .

Infliximab has also been analyzed for its benefit in treating various forms of retinal vasculitis in a retrospective study from a single center in Boston ${ }^{34}$. Using an initial dose of $5 \mathrm{mg} / \mathrm{kg}, 88 \%$ of treated patients were reported to be in remission with this therapy.

Reports on the efficacy of rituximab for retinal vasculitis are sparse. One case report noted resolution of retinal vasculitis after rituximab therapy in a patient who had eosinophilic GPA $^{5}$ and a small treatment study of 20 patients suggested that rituximab may be efficacious in patients with Behçet's. ${ }^{35}$ Rituximab has also shown some success in several 
case reports of patients with SLE and retinal vasculitis. Hickman et al reported the success of rituximab therapy $1 \mathrm{~g}$ intravenously $\times 2$, two weeks apart, in a patient with severe SLEassociated retinal vasculitis that was refractory to cyclophosphamide and intravenous methylprednisolone ${ }^{36}$. Donnithorne et al reported on successful use of rituximab with cyclophosphamide in two female pediatric SLE patients 37 .

Subcutaneously injected alpha interferon has been a successful alternative for therapy of Behçet's disease. This was supported in a study from Korea, but this was based on an experience with only five patients. ${ }^{38}$ All patients experienced some benefit after treatment with alpha interferon with 3 patients achieving a complete response. Visual acuity did not improve in the majority of patients, presumably due to irreversible damage. In contrast, a study of 72 patients from the UK revealed disappointing results. In this study, the addition of pegylated interferon-alpha-2b did not result in an overall reduction in corticosteroid or immunosuppressant dose although a post-hoc analysis showed that patients on corticosteroids at baseline had an improved quality of life and decreased corticosteroid dose at one year ${ }^{39}$. Proponents of alpha interferon as therapy for Behçet's argue that it is as effective as TNF inhibition, that it avoids the risk of immunosuppression, and that it is capable of inducing long term remission. Those who advocate for TNF inhibition point out that this approach to therapy often improves the sense of well-being in contrast to the flu like symptoms engendered by interferon therapy. Inhibition of TNF is probably more effective than interferon for non-ocular manifestations of Behçet's disease. And in addition to flu like symptoms, interferon therapy is associated with a variety of side effects including depression and immune mediated disease such as thyroiditis or even uveitis. Paradoxically inhibiting type I interferon is a promising mode of therapy for systemic lupus erythematosus or dermatomyositis. Just as administering type I interferon can be therapeutic and blocking type I interferon can be therapeutic, IL-17 demonstrates a similarly surprising result. In an animal model of $\mathrm{T}$ cell mediated uveitis, either giving IL-17 ${ }^{40}$ or blocking IL-17 ${ }^{41}$ can be efficacious. This suggests that the immune system seeks homeostatic balance and perturbations in either direction could be therapeutic. An alternative argument is that immune system diseases should be viewed as a spectrum based on immune-pathogenesis with, for instance, multiple sclerosis at one end and rheumatoid arthritis at another. By this argument, successful targeting of a cytokine in one immunological disease should not always extrapolate to the right target in another immune-mediated disease.

\section{CONCLUSIONS}

Retinal vasculitis is usually identified on the basis of perivascular infiltrates or leakage of dye on a fluorescein angiogram in a patient with other evidence for intraocular inflammation and without another cause of retinal vascular disease. The vast majority of patients with retinal vasculitis do not have a systemic vasculitis. Genetic causes of retinal vascular abnormalities are being identified. Behçet's disease is often associated with retinal vasculitis. Monoclonal antibodies that neutralize TNF are extremely effective in treating Behçet's disease. 


\section{Acknowledgements}

We are indebted to Steve Planck who helped with the formatting of this manuscript.

Financial support: This work was supported by NIH Grants K to CAH, K08 EY022948 to PL, and NEI Core Grant P30 EY010572. Additional support was received from Research to Prevent Blindness, the Stan and Madelle Rosenfeld Family Trust, and the William and Mary Bauman Foundation. PL has a career development award from Research to Prevent Blindness.

\section{References}

1. Sigler EJ, Grosso A. Idiopathic Retinal Vasculitis, Aneurysms, and Neuroretinitis Syndrome. Retina. 2015 Epub ahead of print.

2. Westland T, Kamphuis S, van Rossum AM, Rothova A. Case report: an 11-year-old girl presenting with asymptomatic retinal vasculitis leading to diagnosis of HIV-1 infection. Aids. 2015; 29(14): 1901-1902. [PubMed: 26372396]

3. Rosenbaum JT, Ku J, Ali A, Choi D, Suhler EB. Patients with retinal vasculitis rarely suffer from systemic vasculitis. Semin Arthritis Rheum. 2012; 41(6):859-865. [PubMed: 22177107]

4. Sandhu RK, Adams T, Sibley C, Suhler EB, Kim DH. Granulomatosis with Polyangiitis (Gpa) Presenting with Frosted Branch Angiitis. Retinal cases \& brief reports. 2015 Epub ahead of print.

5. Najem CE, Yadav R, Carlson E. Successful use of Rituximab in a patient with recalcitrant multisystemic eosinophilic granulomatosis with polyangiitis. BMJ case reports. 2015:2015.

6. Rosenbaum JT, Roman-Goldstein S, Lindquist GR, Rosenbaum RB. Uveitis and central nervous system vasculitis. J. Rheumatol. 1998; 25(3):593-597. [PubMed: 9517787]

7. Bassuk AG, Yeh S, Wu S, et al. Structural modeling of a novel CAPN5 mutation that causes uveitis and neovascular retinal detachment. PLoS One. 2015; 10(4):e0122352. [PubMed: 25856303]

8*. Wert KJ, Bassuk AG, Wu WH, et al. CAPN5 mutation in hereditary uveitis: the R243L mutation increases calpain catalytic activity and triggers intraocular inflammation in a mouse model. Human molecular genetics. 2015; 24(16):4584-4598. [PubMed: 25994508] Further elucidation of a genetic cause of uveitis.

9*. DiFrancesco JC, Novara F, Zuffardi O, et al. TREX1 C-terminal frameshift mutations in the systemic variant of retinal vasculopathy with cerebral leukodystrophy. Neurological sciences. 2015; 36(2):323-330. [PubMed: 25213617] Further implication of TREX1 in multiorgan vasculopathy.

10. Lee-Kirsch MA, Gong M, Chowdhury D, et al. Mutations in the gene encoding the $3^{\prime}-5^{\prime}$ DNA exonuclease TREX1 are associated with systemic lupus erythematosus. Nat Genet. 2007; 39(9): 1065-1067. [PubMed: 17660818]

11. Crow YJ, Hayward BE, Parmar R, et al. Mutations in the gene encoding the $3^{\prime}-5^{\prime}$ DNA exonuclease TREX1 cause Aicardi-Goutieres syndrome at the AGS1 locus. Nat Genet. 2006; 38(8):917-920. [PubMed: 16845398]

12**. Zhou Q, Wang H, Schwartz DM, et al. Loss-of-function mutations in TNFAIP3 leading to A20 haploinsufficiency cause an early-onset autoinflammatory disease. Nat Genet. 2016; 48(1):67-73. [PubMed: 26642243] The syndrome has Behcet's-like features including chorioretinal scarring and macular fibrosis due to retinal vasculitis.

13. Rani PK, Chhablani J, Bhargava A. Frosted Branch Angiitis in a Patient Coinfected With Dengue Hemorrhagic Fever and Malaria. JAMA Ophthalmol. 2015; 133(6):e1568. [PubMed: 26067694]

14*. Witkin AJ, Shah AR, Engstrom RE, et al. Postoperative Hemorrhagic Occlusive Retinal Vasculitis: Expanding the Clinical Spectrum and Possible Association with Vancomycin. Ophthalmology. 2015; 122(7):1438-1451. [PubMed: 25886796] Intravitreal injection of vancomycin is a suspected cause of a severe retinal vasculitis.

15. Freitas-Neto CA, Boonsopon S, Dhanireddy S, Maghsoudlou A, Silpa-Archa S, Foster CS. Birdshot retinochoroidopathy review. Arquivos brasileiros de oftalmologia. 2015; 78(1):56-61. [PubMed: 25714542] 
16. Tanaka R, Takamoto M, Komae K, Ohtomo K, Fujino Y, Kaburaki T. Clinical features of psoriatic uveitis in Japanese patients. Graefes Arch. Clin. Exp. Ophthalmol. 2015; 253(7):1175-1180. [PubMed: 25725620]

17. Rajpal, Singh UB, Mohapatra S, Wagh VK, Porwal C, Kaushik A. Association of Mycobacterium tuberculosis in the causation of Eales' disease: an institutional experience. Indian journal of medical microbiology. 2015; 33(Suppl):43-45.

18. Shulman S, Kramer M, Amer R, et al. Characteristics and Long-Term Outcome of Patients with Noninfectious Retinal Vasculitis. Retina. 2015; 35(12):2633-2640. [PubMed: 26035402]

19*. Davatchi F, Chams-Davatchi C, Shams H, et al. Adult Behcet's disease in Iran: analysis of 6075 patients. International journal of rheumatic diseases. 2015 A very large database on patients with Behcet's disease; Epub ahead of print.

20*. Arevalo JF, Lasave AF, Al Jindan MY, et al. Uveitis in Behcet disease in a tertiary center over 25 years: the KKESH Uveitis Survey Study Group. Am J Ophthalmol. 2015; 159(1):177-184. e171172. [PubMed: 25448998] A Saudi Arabian perspective on Behcet's disease.

$21 *$. Ali A, Ku JH, Suhler EB, Choi D, Rosenbaum JT. The course of retinal vasculitis. Br. J. Ophthalmol. 2014; 98(6):785-789. [PubMed: 24511084] One of three papers from our group which has characterized retinal vasculitis from a rheumatologic perspective.

22. Birnbaum AD, Fawzi AA, Rademaker A, Goldstein DA. Correlation between clinical signs and optical coherence tomography with enhanced depth imaging findings in patients with birdshot chorioretinopathy. JAMA Ophthalmol. 2014; 132(8):929-935. [PubMed: 24831429]

23. Errera MH, Coisy S, Fardeau C, et al. Retinal vasculitis imaging by adaptive optics. Ophthalmology. 2014; 121(6):1311-1312. e1312. [PubMed: 24576889]

24. Mesquida M, Llorenc V, Fontenla JR, Navarro MJ, Adan A. Use of ultra-wide-field retinal imaging in the management of active Behcet retinal vasculitis. Retina. 2014; 34(10):2121-2127. [PubMed: 24946103]

25*. Campbell JP, Beardsley RM, Palejwala NV, et al. Peripheral vascular leakage in uveitis: clinical and angiographic findings. Ophthalmology. 2015; 122(6):1269-1270. [PubMed: 25769846] Peripheral vascular leakage is associated with poor clinical control and cystoid macular edema.

26. Gul A, Tugal-Tutkun I, Dinarello CA, et al. Interleukin-1beta-regulating antibody XOMA 052 (gevokizumab) in the treatment of acute exacerbations of resistant uveitis of Behcet's disease: an open-label pilot study. Annals of the rheumatic diseases. 2012; 71(4):563-566. [PubMed: 22084392]

27. Hueber W, Patel DD, Dryja T, et al. Effects of AIN457, a fully human antibody to interleukin-17A, on psoriasis, rheumatoid arthritis, and uveitis. Science translational medicine. 2010; 2(52):52ra72.

28. Dick AD, Tugal-Tutkun I, Foster S, et al. Secukinumab in the treatment of noninfectious uveitis: results of three randomized, controlled clinical trials. Ophthalmology. 2013; 120(4):777-787. [PubMed: 23290985]

29. Springer TA. Adhesion receptors of the immune system. Nature. 1990; 346:425-433. [PubMed: 1974032]

30. Levy-Clarke G, Jabs DA, Read RW, Rosenbaum JT, Vitale A, Van Gelder RN. Expert panel recommendations for the use of anti-tumor necrosis factor biologic agents in patients with ocular inflammatory disorders. Ophthalmology. 2014; 121(3):785-796. e783. [PubMed: 24359625]

31. Calvo-Río V, Blanco R, Beltrán E, et al. Anti-TNF-a therapy in patients with refractory uveitis due to Behçet's disease: a 1-year follow-up study of 124 patients. Rheumatology. 2014; 53(12):22232231. [PubMed: 24996907]

$32 *$. Takeuchi M, Kezuka T, Sugita S, et al. Evaluation of the long-term efficacy and safety of infliximab treatment for uveitis in Behcet's disease: a multicenter study. Ophthalmology. 2014; 121(10):1877-1884. [PubMed: 24950593] The largest cohort study to date demonstrating a positive clinical response to TNF inhibition in Behcet's Disease.

33. Vallet H, Riviere S, Sanna A, et al. Efficacy of anti-TNF alpha in severe and/or refractory Behcet's disease: Multicenter study of 124 patients. J Autoimmun. 2015; 62:67-74. [PubMed: 26162757]

34. Sharma PK, Markov GT, Bajwa A, Foster CS. Long-Term Efficacy of Systemic Infliximab in Recalcitrant Retinal Vasculitis. Retina. 2015; 35(12):2641-2646. [PubMed: 26200508] 
35. Davatchi F, Shams H, Rezaipoor M, et al. Rituximab in intractable ocular lesions of Behcet's disease; randomized single-blind control study (pilot study). International journal of rheumatic diseases. 2010; 13(3):246-252. [PubMed: 20704622]

36. Hickman RA, Denniston AK, Yee CS, Toescu V, Murray PI, Gordon C. Bilateral retinal vasculitis in a patient with systemic lupus erythematosus and its remission with rituximab therapy. Lupus. 2010; 19(3):327-329. [PubMed: 19900982]

37. Donnithorne KJ, Read RW, Lowe R, Weiser P, Cron RQ, Beukelman T. Retinal vasculitis in two pediatric patients with systemic lupus erythematosus: a case report. Pediatric rheumatology online journal. 2013; 11(1):25. [PubMed: 23734963]

38. Park JY, Chung YR, Lee K, Song JH, Lee ES. Clinical Experience of Interferon Alfa-2a Treatment for Refractory Uveitis in Behcet's Disease. Yonsei medical journal. 2015; 56(4):1158-1162. [PubMed: 26069144]

39*. Lightman S, Taylor SR, Bunce C, et al. Pegylated interferon-alpha-2b reduces corticosteroid requirement in patients with Behcet's disease with upregulation of circulating regulatory $\mathrm{T}$ cells and reduction of Th17. Annals of the rheumatic diseases. 2015; 74(6):1138-1144. [PubMed: 25269831] Although interferon treatment did not reach its primary endpoint, a secondary analysis showed benefit and it had potentially beneficial effects on Th17 T cells and regulatory $\mathrm{T}$ cells.

40. Ke Y, Liu K, Huang GQ, et al. Anti-inflammatory role of IL-17 in experimental autoimmune uveitis. J. Immunol. 2009; 182(5):3183-3190. [PubMed: 19234216]

41. Luger D, Silver PB, Tang J, et al. Either a Th17 or a Th1 effector response can drive autoimmunity: conditions of disease induction affect dominant effector category. J. Exp. Med. 2008; 205(4):799810. [PubMed: 18391061] 


\section{Key Points}

1) The term, "vasculitis", often has vastly different implications for an ophthalmologist compared to a rheumatologist.

2)

Retinal vascular disease can be genetic secondary to mutations in CAPN5, TREX1, or TNFAIP3.

2)

New imaging modalities can better detect retinal vasculitis, but the clinical significance of peripheral retinal vascular leakage is controversial.

3)

Monoclonal antibodies against TNF are effective in treating Behçet's disease, a well recognized cause of retinal vasculitis, while antibodies to IL-17A or IL-1 beta have been disappointing for this indication. 


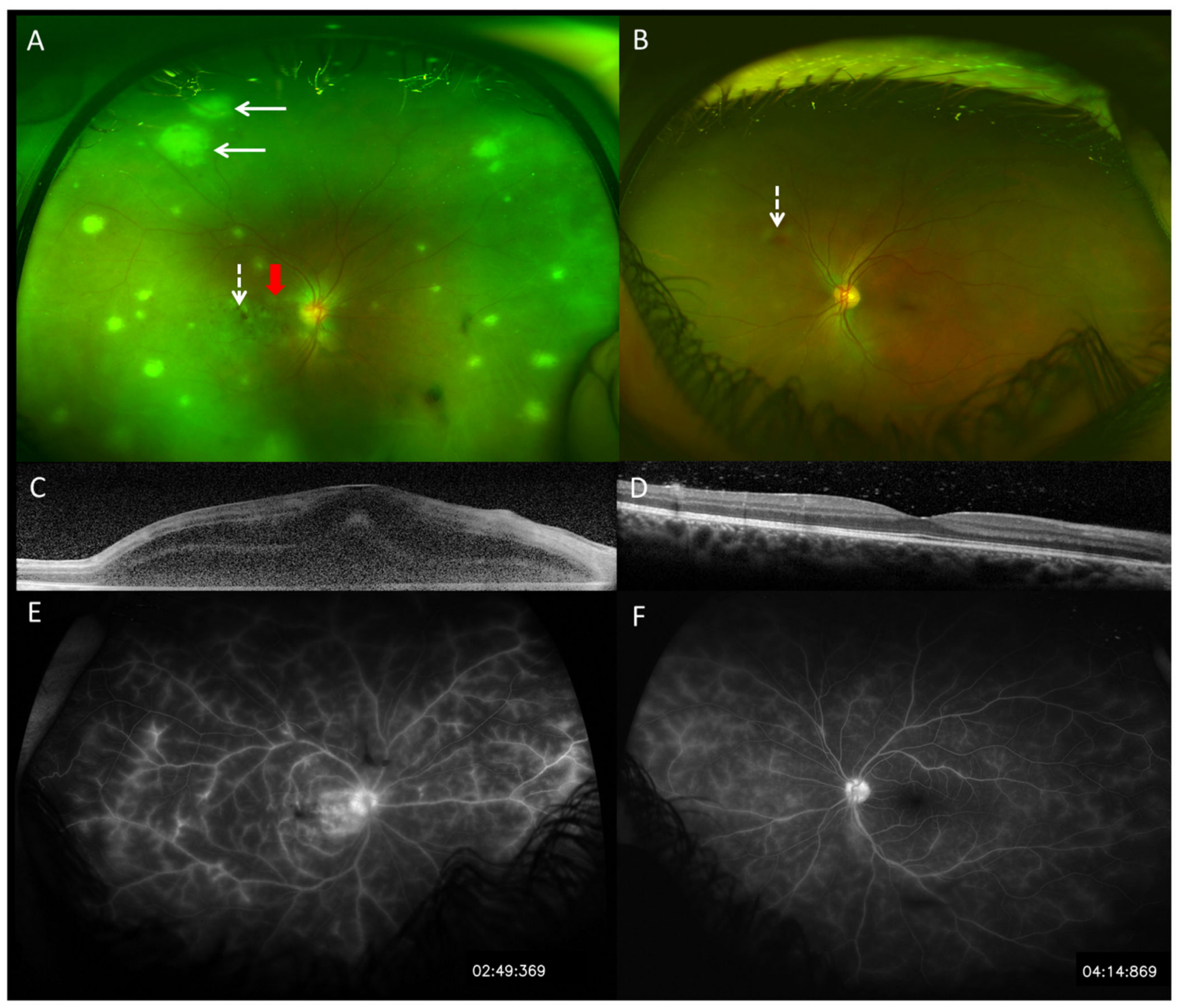

Figure 1.

Fundus imaging, optical coherence tomography(OCT), and ultra widefield fluorescein angiography findings upon initial presentation of a 25 year-old Saudi male diagnosed with Behçet's disease. Fundus imaging of the right (A) and left (B) eyes reveal multifocal retinal infiltrates (solid white arrows), cotton wool spots (red arrow), and intraretinal hemorrhages (dotted white arrow) in the right eye > left eye. OCT reveals cystoid macular edema and a serous retinal detachment in the right eye (C), and a normal left eye (D). Ultra wide field imaging demonstrates diffuse retinal vascular leakage in both eyes, greater in the right (E) than the left $(\mathrm{F})$. 

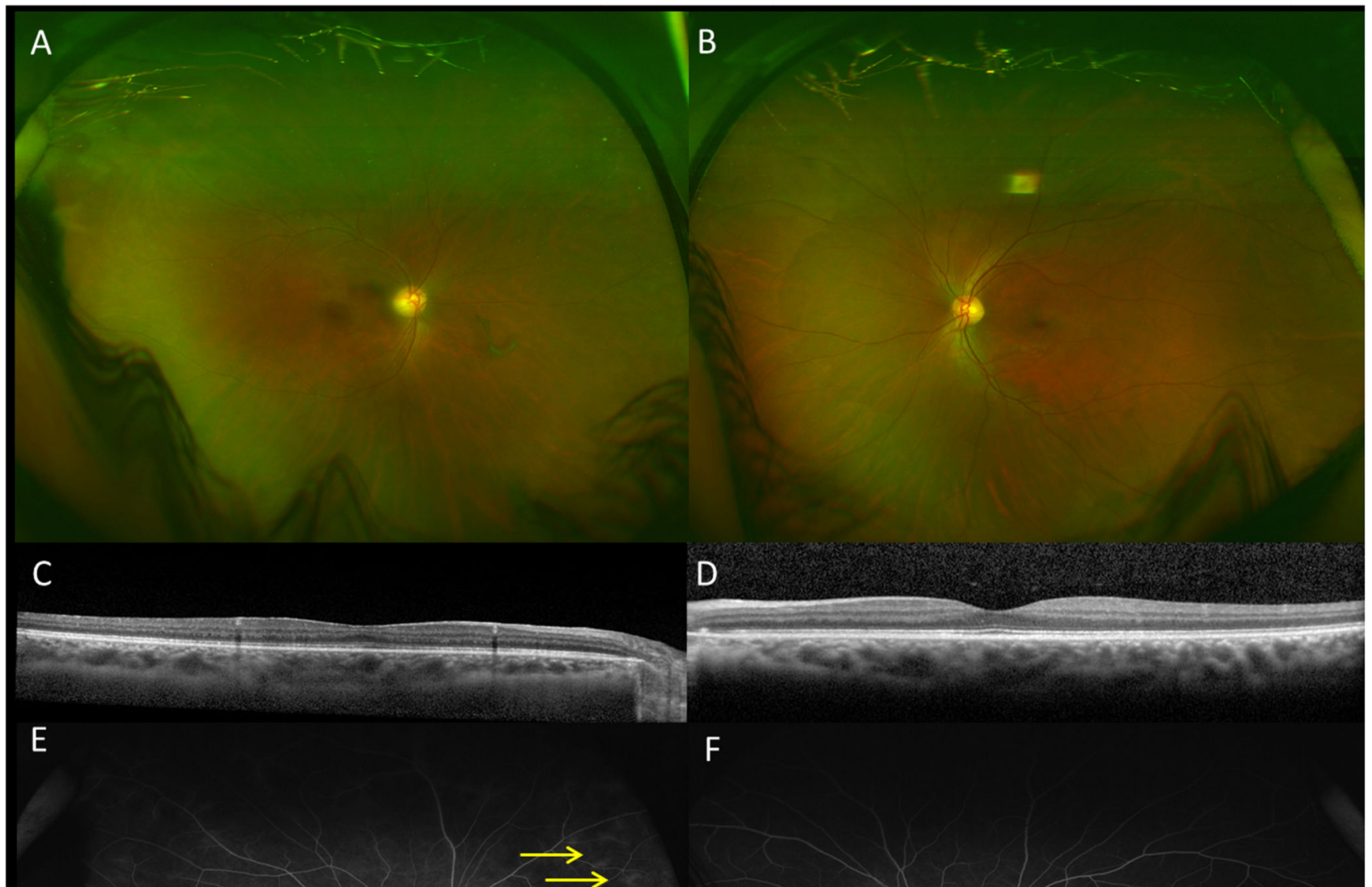

Figure 2.

Improvement in fundus imaging, optical coherence tomography (OCT), and ultra widefield fluorescein angiography findings after treatment with prednisone, azathioprine, and infliximab in Behçet's disease. Fundus imaging of the right (A) and left (B) eyes reveal resolution of intraretinal infilrates and hemorrhages. OCT reveals resolution of cystoid macular edema and serous retinal detachment in the right eye $(\mathrm{C})$, and normal OCT on the left eye (D). ultra widefield imaging demonstrates residual retinal vascular leakage in the right eye (yellow arrows) (E) and no retinal vascular leakage in the left eye (F). 


\section{Table 1}

Potential etiology based on retinal arteriole- or venule-predominance of retinal vasculitis.

\begin{tabular}{|l|l|l|}
\hline Primarily retinal arteritis & Primarily retinal phlebitis & Arteritis and phlebitis \\
\hline $\begin{array}{l}\text { Systemic lupus } \\
\text { erythematosus }\end{array}$ & Sarcoidosis & Relapsing polychondritis \\
\hline Polyarteritis nodosa & Multiple sclerosis & GPA \\
\hline Syphilis & Pars planitis & Crohn's disease/UC \\
\hline Toxoplasmosis & Behçet's disease & Frosted branch angiitis \\
\hline HSV/VZV & Birdshot chorioretinopathy & Tuberculosis \\
\hline IRVAN & $\begin{array}{l}\text { HIV associated retinal } \\
\text { vasculitis }\end{array}$ & \\
\hline Churg-Strauss syndrome* & Eales disease & \\
\hline Susac syndrome & & \\
\hline
\end{tabular}

HSV: herpes simplex virus; VZV: varicella zoster virus; IRVAN: idiopathic retinal vasculitis, aneurysms, and neuroretinitis; HIV: human immunodeficiency virus; GPA: granulomatosis with polyangiitis; UC: ulcerative colitis.

These diseases are rare causes of retinal vasculitis so characterization is based on a small number of descriptions. 